\title{
Angiogenic and angiostatic factors in renal scleroderma-associated vasculopathy is
}

\author{
Antonietta Gigante ${ }^{\mathrm{a}, *}$, Luca Navarini ${ }^{\mathrm{b}}$, Domenico Margiotta ${ }^{\mathrm{b}}$, Antonio Amoroso $^{\mathrm{a}}$, Biagio Barbano $^{\mathrm{a}}$, \\ Rosario Cianci ${ }^{\mathrm{a}}$, Antonella Afeltra ${ }^{\mathrm{b}}$, Edoardo Rosato ${ }^{\mathrm{a}}$ \\ a Department of Clinical Medicine, Sapienza University of Rome, Rome, Italy \\ b Department of Immuno-Rheumatology Unit, Campus Bio-Medico University of Rome, Rome, Italy
}

\section{A R T I C L E I N F O}

\section{Article history:}

Received 28 March 2017

Revised 7 June 2017

Accepted 7 June 2017

Available online 8 June 2017

\section{Keywords:}

Systemic sclerosis

Renal resistive index

VEGF

Endostatin

Angiogenesis

\begin{abstract}
A B S T R A C T
Background: The angiogenesis in systemic sclerosis (SSc) is impaired. An imbalance of pro-angiogenic factors and angiogenesis inhibitors has been implicated in the progression of peripheral microvascular damage, defective vascular repair and fibrosis. Intrarenal resistance index are considered markers of renal vasculopathy. The aim of the study is to evaluate angiogenic and angiostatic factors (VEGF and endostatin) in SSc patients and to correlate with intrarenal hemodynamic parameters.

Methods: 91 SSc patients were enrolled in this study. Serum VEGF and endostatin levels were determined. All patients underwent a renal Doppler ultrasound

Results: A significant positive correlation was observed between endostatin and renal Doppler parameters $(p<0.0001)$. A negative correlation was observed between serum levels of endostatin and eGFR $(p<0.01)$. In SSc patients with high resistive index, serum levels of endostatin were significantly $(p<0.01)$ higher than in SSc patients with normal resistive index. The serum levels of endostatin significantly increased with progression of nailfold videocapillaroscopy damage $(p<0.01)$ and were significantly $(p<0.05)$ higher in SSc patients with digital ulcers than in SSc patients without digital ulcers.

Conclusion: This is the first study that assess in SSc patients intrarenal hemodynamic parameters and endostatin. In SSc patients, endostatin represents a marker of renal scleroderma-associated vasculopathy.
\end{abstract}

(c) 2017 Elsevier Inc. All rights reserved.

\section{Background}

Systemic sclerosis (SSc) is a connective tissue disease characterized by vasculopathy, collagen deposition and fibrosis in the skin and internal organs. In SSc the endothelial dysfunction is the most important feature and involves both the macro and microvasculature (Campbell and LeRoy, 1975).

The progressive derangement of the microcirculation leads to a state of chronic tissue hypoxia that stimulates the release of angiogenic growth factors such as vascular endothelial growth factors (VEGF).

\footnotetext{
Abbreviations: ACE-I, angiotensin-converting enzyme inhibitors; CKD, chronic kidney disease; DAI, Disease Activity Index; dcSSc, diffuse cutaneous SSc; DSS, Disease Severity Scale; EDV, end diastolic velocity; GFR, glomerular filtrate rate; HC, healthy controls; lcSSc, limited cutaneous SSc; mRSS, Modified Rodnan total skin score; NVC, Nailfold videocapillaroscopy; RI, resistive index; PAH, Pulmonary arterial hypertension; PI, pulsatile index; PSV, peak systolic velocity; sCr, serum creatinine; SD, standard deviation; S/D, systolic/diastolic ratio; SSc, Systemic sclerosis; UA, serum uric acid; VEGF, vascular endothelial growth factors.

¿ No support/fund and/or conflict of interest to be declared.

* Corresponding author at: Department of Clinical Medicine, Sapienza University of Rome, Viale dell'Università, 37, 00185 Rome, Italy.

E-mail address: antonietta_gigante@yahoo.it (A. Gigante).
}

Conversely, the inhibitors of angiogenesis are represented by endostatin and angiostatin (Hummers et al., 2009).

Several forms of renal damage are recognized in patients with SSc, from asymptomatic reduction of glomerular filtrate rate (GFR), reduced renal functional reserve, microalbuminuria and increased intrarenal arterial stiffness to renal scleroderma crisis (Shanmugam and Steen, 2010).

The association of renal dysfunction and vasculopathy is common in SSc and usually exhibits a benign course.

Regardless of SSc, chronic kidney disease (CKD) presents a defective angiogenesis with high levels of endostatin and reduced VEGF (Futrakul et al., 2008). The aim of the study is to evaluate two angiogenetic and angiostatic factors, such as VEGF and endostatin, in SSc patients and to correlate with intrarenal hemodynamic parameter.

\section{Methods}

91 patients ( 80 female and 11 male; mean age $54.4 \pm 13.3$ years) fulfilling the American College of Rheumatology/European League criteria for classification of SSc were enrolled in this study (van den Hoogen et al., 2013). 
44 patients had limited cutaneous SSc (lcSSc) and 47 presented diffuse cutaneous SSc (dcSSc) as defined by LeRoy et al. (1988).

All SSc patients underwent treatment with calcium channel blockers (nifedipine $30 \mathrm{mg} /$ day). None of patients were treated with immunosuppressive agents (e.g. cyclophosphamide or mycophenolate mofetil), angiotensin-converting enzyme inhibitors (ACE-I), angiotensin II receptor blockers, diuretics or xanthine oxidase inhibitors.

Patients with urinary tract infections, abnormal urinary sediment, glomerulonephritis, acute and chronic kidney disease, scleroderma renal crisis, kidney stones, anti-phospholipid-associated nephropathy, diabetes, cardiovascular diseases such as hypertension, myocardial infarction, arrhythmias, heart failure, hyperlipidaemia, coagulopathy, obesity or smokers were excluded.

48 healthy controls (HC) (42 female and 6 male; mean age $49.7 \pm$ 15.3 years) were also enrolled in this study.

The subjects' written consent was obtained according to the Declaration of Helsinki and the study was approved by ethics committee of Sapienza University (IRB approval 377/2014).

\subsection{Laboratory parameters}

Laboratory investigations included serum creatinine $(\mathrm{sCr})$ (normal range: $0.5-0.9 \mathrm{mg} / \mathrm{dl}$ ), blood urea nitrogen (normal range: $10.20-$ $49.80 \mathrm{mg} / \mathrm{dl}$ ), serum uric acid (UA) (normal range: $3.40-7.20 \mathrm{mg} / \mathrm{dl}$ ), urinalysis and $24 \mathrm{~h}$ proteinuria. $\mathrm{sCr}$ was measured using a Jaffe alkaline picrate assay (Abbott Aeroset analyser) (Wetzels et al., 2007). Serum UA was measured with an automatic analyzer (7700 series; Hitachi, Tokyo, Japan).

\subsection{Calculation of GFR}

GFR was calculated using the CKD-EPI equation, already validated in SSc (Gigante et al., 2012), expressed as a single equation: GFR $=141 \times$ $\min (\mathrm{sCr} / \mathrm{k}, 1) \alpha \times \max (\mathrm{sCr} / \mathrm{k}, 1)-1.209 \times 0.993$ Age $\times 1.018$ (if female) $\times 1.159$ (if black), where $\mathrm{k}$ is 0.7 for females and 0.9 for males, $\alpha$ is -0.329 for females and -0.411 for males, min indicates the minimum of $\mathrm{sCr} / \mathrm{k}$ or 1 and max indicates the maximum of $\mathrm{sCr} / \mathrm{k}$ or 1 (Levey et al., 2009).

\subsection{Nailfold videocapillaroscopy}

Nailfold videocapillaroscopy (NVC) was performed with a videocapillaroscope (Pinnacle Studio Version 8) equipped with a 500 $x$ optical probe. The nailfold of the second, third, fourth and fifth finger was examined in each patient. According to Cutolo et al., patterns identified within the "SSc pattern" include: early, active and late (Cutolo et al., 2006).

\subsection{Clinical assessment}

Modified Rodnan total skin score (mRSS) was chosen as the most used method to assess skin induration in SSc. It is determined at a standardized location of 17 different sites of the body with a standardized pinching method and it is scored from 0 to 3 (Clements et al., 1995).

Disease activity in SSc was measured using Disease Activity Index (DAI), which consists of 10 weighted variables: total skin score $>14$, scleroderma, digital necrosis, arthritis, total lung capacity $<80 \%$, erythrocyte sedimentation rate $($ ESR) $>30$, hypocomplementemia and change in cardiopulmonary, skin and vascular symptoms in the past month (Valentini et al., 2001).

Disease severity was measured by Medsger Disease Severity Scale (DSS). The original scale assessed disease severity in 9 organs or systems, namely general health, peripheral vascular, skin, joint/tendon, muscle and gastrointestinal tract, lungs, heart and kidneys. Each organ/system is scored separately from 0 to 4 depending on whether there is no, mild, moderate, severe or end-stage involvement (Medsger et al., 1999).

\subsection{ELISA for VEGF and endostatin}

Serum VEGF levels were determined in SSc patients by commercial ELISA kit (Human VEGF, Quantikine ELISA, R\&D Systems, Minneapolis $\mathrm{MN}$ ), with a sensitivity of $9 \mathrm{pg} / \mathrm{ml}$ and an assay range of 31.2$2000 \mathrm{pg} / \mathrm{ml}$, according to the instructions provided by the manufacturer. After a 50-fold diluition, serum endostatin levels were determined in SSc patients by commercial ELISA kit (Human Endostatin, Quantikine ELISA, R\&D Systems, Minneapolis MN), with a sensitivity of $0.063 \mathrm{ng} / \mathrm{ml}$ and an assay range of $0.3-10 \mathrm{ng} / \mathrm{ml}$, according to the instructions provided by the manufacturer.

\subsection{Doppler ultrasound}

SSc patients were placed for at least $15 \mathrm{~min}$ before the Doppler ultrasound examination in a temperature-controlled room at $22 \pm 0.4{ }^{\circ} \mathrm{C}$. The size of the left and right kidneys and the flow in the aorta and renal arteries were evaluated to detect a morphologic abnormality or renal artery stenosis. During the measurements, the patients were supine and held their breath. Doppler ultrasound examinations were performed by the same senior nephrologist blinded to the clinical features of the patient. Renal Doppler ultrasound was performed using a Toshiba Aplio Ultrasound System SSA-790 equipped with a convex 3.5-MHz probe. Renal Doppler flow was obtained in 3 different interlobar arteries of both kidneys (mesorenal, superior, and inferior pole), guided by color-flow mapping. The Doppler gate width was kept small, and the angle of insonation was maintained at $<60^{\circ}$. We used an anterior approach for detecting the renal artery origin and an oblique, lateral approach for the intermediate tract and intrarenal vessels. No aliasing was allowed in the interlobar arteries while the following parameters were measured: peak systolic velocity (PSV), end diastolic velocity (EDV), resistive index (RI), pulsatile index (PI), and systolic/diastolic ratio (S/D). RI was calculated as (peak systolic frequency shift - minimum diastolic frequency shift) / peak systolic frequency shift and the PI was calculated as (peak systolic frequency shift / minimum diastolic frequency shift) / mean frequency shift. The PSV and EDV are expressed as $\mathrm{cm} / \mathrm{s}$. The mean value of 3 measurements of interlobar arteries from each kidney was calculated. For each patient, Doppler ultrasound parameters were calculated as the mean of measurements of both kidneys. While performing the renal Doppler examination, the operator evaluated the heart function. The Doppler examination was not performed in SSc patients with arrhythmia or tachycardia or bradycardia. Weighted kappa was used to evaluate the intra rater reliability by the same observer. The kappa values for RI and S/D were 0.971 and 0.975 , respectively. The intrapatient coefficient of variation for RI and S/D measurement was $1.3 \%$ and $1.4 \%$, respectively. Calcium-channel blocker therapy was discontinued $72 \mathrm{~h}$ before the Doppler ultrasound (Rosato et al., 2012). The mean references value for normal RI in adults is determinate to be $0.60 \pm 0.10$, with 0.70 as upper limit of normal (Parolini et al., 2009).

Patients receiving iloprost therapy underwent Doppler examination the day before the next infusion.

\subsection{Statistical analysis}

The results were expressed as mean and standard deviation (SD) or median and range, as appropriate. Commercially software (SPSS version 23.0) was used for statistical analysis. The coefficient of skewness and the coefficient of kurtosis were used to evaluate the normal distribution of data. Multiple regression analysis was applied to evaluate the relationship between Doppler indices of intrarenal arterial stiffness and serum levels of VEGF and endostatin and the demographic and clinical features (age, duration of disease, mRSS, DAI, DSS) and internal organ 
damage indices (creatinine, CKD-EPI, PAPs, DLCO, FVC). Pearson product-moment or Spearman correlation coefficient ( $r$ ) was used to test for bivariate analysis. Group comparisons were made by Student's unpaired 2-tailed $t$-test or the Kruskal-Wallis test, as appropriate. p-Values $<0.05$ were considered significant.

\section{Results}

The SSc patients' epidemiological and clinical features are showed in Table 1. The Doppler indices of intrarenal arterial stiffness were significantly ( $p<0.0001$ ) higher in SSc patients than HC. No significant difference of creatinine, serum uric acid and GFR was observed between SSc patients and HC (Table 2). The medium values of serum VEGF and endostatin were $283.4 \pm 250.8 \mathrm{pg} / \mathrm{ml}$ and $118.1 \pm 38.4 \mathrm{ng} / \mathrm{ml}$, respectively.

A significant positive correlation was observed between endostatin and PI $(\mathrm{r}=0.35, \mathrm{p}<0.0001)$, RI $(\mathrm{r}=0.38, \mathrm{p}<0.0001)$ and S/D ratio $(r=0.35, p<0.0001)$ (Fig. 1). A negative correlation was observed between serum levels of endostatin and eGFR $(r=-0.30, p<0.01)$. No significant $(p>0.05)$ correlation was observed between VEGF and eGFR.

In the multiple regression analysis we analyzed Doppler indices of intrarenal arterial stiffness as dependent variables (Table 3). In the analysis we observed a correlation between Doppler indices of intrarenal arterial stiffness and age, eGFR, evaluated by CKD-EPI, and endostatin. Only PI demonstrated a significant positive correlation with serum levels of VEGF $(r=0.31, p<0.01)$. A significant positive correlation was observed between age and PI $(r=0.46, p<0.0001)$, RI $(r=0.57$, $\mathrm{p}<0.0001)$ and $\mathrm{S} / \mathrm{D}$ ratio $(\mathrm{r}=0.56, \mathrm{p}<0.0001)$. A significant negative correlation was observed between eGFR and PI $(\mathrm{r}=-0.18, \mathrm{p}<0.05)$, $\mathrm{RI}(\mathrm{r}=-0.24, \mathrm{p}<0.05)$ and $\mathrm{S} / \mathrm{D}$ ratio $(\mathrm{r}=-0.23, \mathrm{p}<0.05)$.

Using a cut-off value of 0.70 for RI, we grouped SSc patients in two subgroups: normal RI $(\leq 0.69)$ and high RI $(\geq 0.70)$. In SSc patient with high RI serum levels of endostatin are significantly $(\mathrm{p}<0.01)$ higher than in SSc patients with normal RI $(129.4 \pm 41.4$ vs $105.3 \pm 30.6)$ (Fig. 2). No significant difference of serum levels of VEGF was observed in SSc patients with normal or high RI.

The serum levels of endostatin $(\mathrm{ng} / \mathrm{ml})$ significantly $(\mathrm{p}<0.01)$ increased with progression of NVC damage: early ( $98.9 \pm 40.9)$, active $(114.7 \pm 35.5)$ and late $(133.9 \pm 33.9)$. The serum levels of VEGF $(\mathrm{pg} / \mathrm{ml})$ did not show any significant $(\mathrm{p}>0.05)$ difference in three capillaroscopic groups of patients: early (274.4 \pm 259.7$)$, active $(268 \pm 221.5)$ and late $(305 \pm 278)$. The serum levels of endostatin $(\mathrm{ng} / \mathrm{ml})$ were significantly $(\mathrm{p}<0.05)$ higher in SSc patients with digital ulcers (DUs) than in SSc patients without DUs (127 \pm 31.8 vs $107 \pm$ 43.4) (Fig. 3). No significant ( $\mathrm{p}>0.05$ ) difference of VEGF ( $\mathrm{pg} / \mathrm{ml}$ ) was observed in SSc with DUs ( $302 \pm 244.8)$ or without DUs (259 \pm 259.2$)$.

Table 1

SSc patients' epidemiological and clinical features.

\begin{tabular}{ll}
\hline Sex (female/male) & $80 / 11$ \\
\hline Age (years) & $54.4 \pm 13.3$ \\
Disease duration (years) & $9 \pm 6$ \\
DAI & $2.8 \pm 2.4$ \\
DSS & $5 \pm 3.2$ \\
dcSSc/lcSSc & $47 / 44$ \\
Digital ulcers history & $51(56)$ \\
SSc-specific autoantibodies & $\mathrm{n}(\%)$ \\
Anti-topoisomerase I & $50(54.9)$ \\
Anti-centromere & $37(40.7)$ \\
None & $4(4.4)$ \\
Capillaroscopic pattern & $\mathrm{n}(\%)$ \\
Early & $22(24.2)$ \\
Active & $35(38.5)$ \\
Late & $34(37.4)$ \\
\hline
\end{tabular}

Disease Activity Index (DAI); Disease Severity Scale (DSS); limited cutaneous SSc (lcSSc); diffuse cutaneous SSc (dcSSc).
Table 2

The mean value of serum UA and sCr, CKD-EPI, Doppler ultrasound indices in all SSc patients.

\begin{tabular}{llll}
\hline & SSc & HC & $\mathrm{p}$ \\
\hline PI & $1.43 \pm 0.29$ & $1.02 \pm 0.16$ & $\mathrm{p}<0.0001$ \\
RI & $0.70 \pm 0.06$ & $0.51 \pm 0.05$ & $\mathrm{p}<0.0001$ \\
S/D & $3.52 \pm 0.79$ & $2.11 \pm 0.23$ & $\mathrm{p}<0.0001$ \\
SCr & $0.74 \pm 0.18$ & $0.68 \pm 0.13$ & $\mathrm{p}>0.05$ \\
CKD-EPI & $94.1 \pm 19$ & $99.3 \pm 9.5$ & $\mathrm{p}>0.05$ \\
Serum UA & $4.22 \pm 1.12$ & $4.14 \pm 0.74$ & $\mathrm{p}>0.05$ \\
IMT & $0.75 \pm 9$ & $0.70 \pm 13$ & $\mathrm{p}>0.05$ \\
Systolic blood pressure (mm Hg) & $112 \pm 9$ & $116 \pm 12$ & $\mathrm{p}>0.05$ \\
Diastolic blood pressure (mm Hg) & $71 \pm 6$ & $75 \pm 8$ & $\mathrm{p}>0.05$ \\
BMI & $23 \pm 3.2$ & $24 \pm 4.2$ & $\mathrm{p}>0.05$ \\
\hline
\end{tabular}

Uric acid (UA); serum creatinine ( $\mathrm{sCr}$ ); Chronic Kidney Disease Epidemiology Collaboration (CKD-EPI); pulsatile index (PI); resistive index (RI); systolic/diastolic ratio (S/D), Intimal media thickness (IMT).

\section{Discussion}

Angiogenesis is characterized by the formation of new blood vessels under control of angiogenesis inducers and inhibitors. It is well known that in course of SSc the angiogenesis is impaired promoting disarrangement of the microcirculation (Hummers et al., 2009).

In our study, serum VEGF and endostatin values are comparable to data in the literature and, in particular, are higher in SSc patients when compared to healthy population (Hummers et al., 2009; Farouk et al., 2013; Hebbar et al., 2000; Distler et al., 2002).

Endostatin shows a positive correlation with intrarenal hemodynamic parameters and, conversely, a negative correlation with eGFR.

Recent studies have suggested that endostatin is able to influence the regulation of the extracellular and metalloproteinase matrix, which may contribute to vascular remodeling in SSc (Peng et al., 2012).

Endostatin is an angiogenic inhibitor derived from type XVIII collagen, which is particularly expressed in the fenestrated endothelium of renal microcirculation (Lin et al., 2016).

In course of SSc elevated endostatin levels correlate with skin sclerosis, cutaneous scars, digital ulcers (Hebbar et al., 2000), giant capillaries in NVC (Distler et al., 2002), pulmonary arterial hypertension (PAH) and SCR (Reiseter et al., 2015).

Reiseter et al. in an observational study have evaluated the serum levels of endostatin and VEGF in unselected cohorts of 298 SSc patients and 162 mixed connective tissue disease patients. Mean levels of endostatin were increased in SSc patients when compared to HC. The most vascular complications in course of disease like PAH and SRC were associated with elevated endostatin levels. Furthermore, endostatin resulted as only predictor of SRC and its high levels showed a strong association with SSc mortality during follow-up. The histopathological features are related to endothelial dysfunction with intimal thickening and fibrotic 'onion-skinning' of the interlobular and arcuate renal arteries. Thus, Reiseter et al. hypothesize that dysregulated angiogenesis may play a role in SRC in association to endostatin in the regulation of matrix metalloproteinases and vascular remodeling (Reiseter et al., 2015).

For this reasons some authors suppose that in SSc, during ischemic manifestations (Hebbar et al., 2000), endostatin is over-expressed and could establish a link between tissue collagen deposition and vascular damage.

Also in CKD (Maeshima and Makino, 2010) and arterial hypertension (Marek-Trzonkowska et al., 2015) there is a dysbalance of angiogenic and angiostatic factors. A simultaneous increase of angiogenic inhibitor and reduction of pro-angiogenic factors occur in hypertensive patients. Furthermore high levels of endostatin may determine microvascular dysfunction with loss of terminal arterioles and capillaries and increasing of peripheral resistance.

Similarly in our study, intrarenal hemodynamic parameters and endostatin can increase in response to ischemic and hypoxic conditions. 

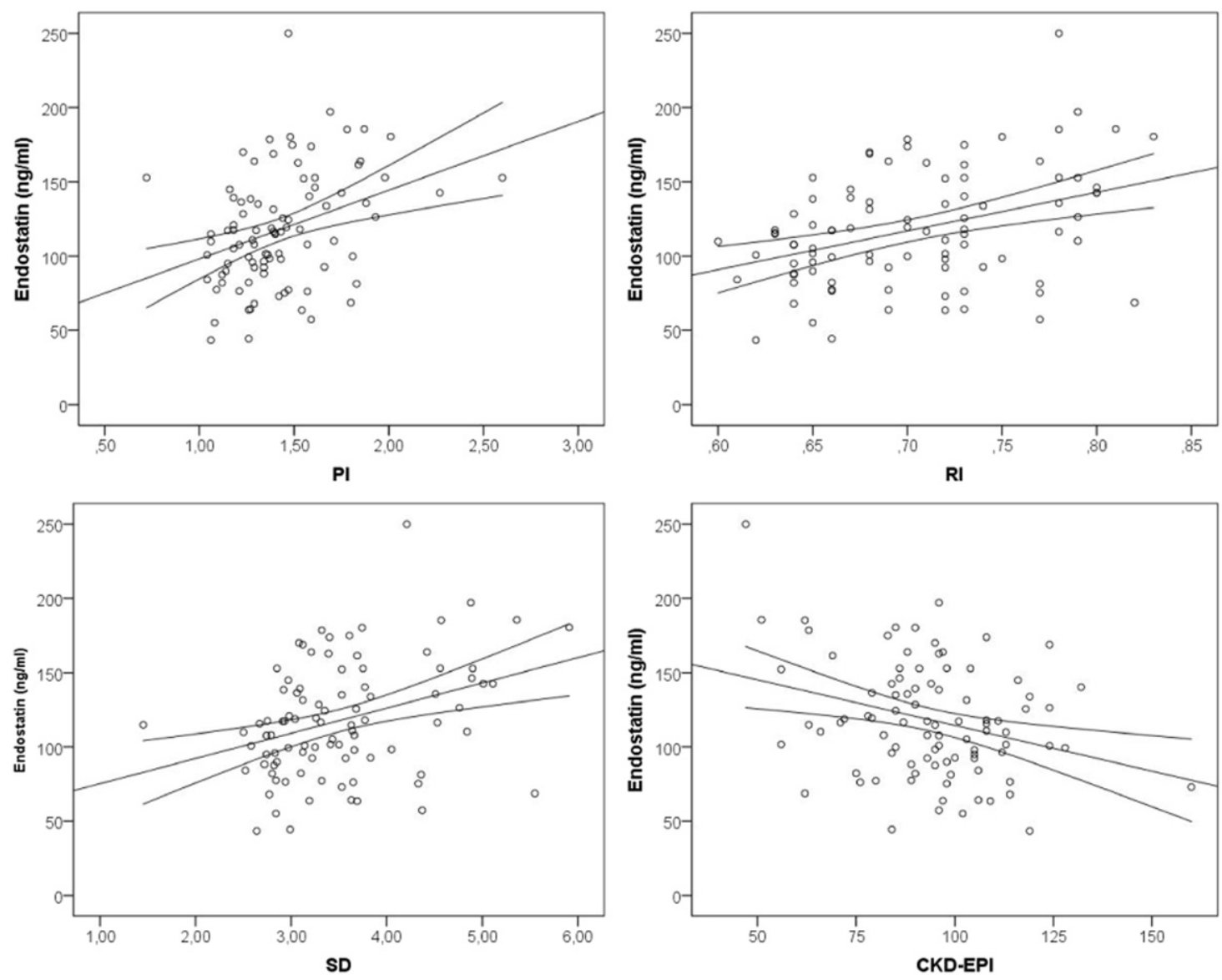

Fig. 1. Correlation between serum levels of endostatin ( $\mathrm{ng} / \mathrm{ml})$ and Doppler indices of intrarenal arterial stiffness or estimated glomerular filtration rate (CKD-EPI).

We suppose that enhanced endostatin reflects a chronic vascular activity in course of renal scleroderma-associated vasculopathy.

Rosato et al. have demonstrated that in the initial phases of SSc renal injury, RI increases and measured GFR is normal or slightly reduced because is more linked to reduced blood inflow to intrarenal vessels than reduced glomerular filtration capacity (Rosato et al., 2012).

Our results show a negative correlation between intrarenal arterial stiffness (PI, RI, and S/D ratio) and eGFR. In fact, eGFR reduction is due to intrarenal arteries blood inflow and to glomerular damage that occurs with renal vascular progression. In a study conducted in elderly patients, endostatin showed a negative correlation with GFR and it was associated with CKD progression (Ruge et al., 2014). Furthermore other studies have demonstrated that in SSc renal vasculopathy, renal functional reserve (Mohamed et al., 2010) is reduced as well as renal

Table 3

Linear regression analysis models of correlations between intrarenal arterial stiffness and other variables of diseases.

\begin{tabular}{llll}
\hline $\begin{array}{l}\text { Doppler indices } \\
\text { (dependent) }\end{array}$ & $\begin{array}{l}\text { Other variables } \\
\text { (independent) }\end{array}$ & $\begin{array}{l}\text { Standardized } \\
\beta \text {-coefficient }\end{array}$ & $\begin{array}{l}\mathrm{p} \\
\text { value }\end{array}$ \\
\hline PI & Age & 0.384 & $<0.05$ \\
& CKD-EPI & 0.247 & $<0.05$ \\
& Endostatin & 0.205 & $<0.05$ \\
VEGF & 0.198 & $<0.05$ \\
RI & Age & 0.477 & $<0.001$ \\
& CKD-EPI & 0.259 & $<0.05$ \\
S/D ratio & Endostatin & 0.228 & $<0.01$ \\
& Age & 0.384 & $<0.05$ \\
& CKD-EPI & 0.247 & $<0.05$ \\
& Endostatin & 0.198 & $<0.05$ \\
\hline
\end{tabular}

Chronic Kidney Disease Epidemiology Collaboration (CKD-EPI); pulsatile index (PI); resistive index (RI); systolic/diastolic ratio (S/D); vascular endothelial growth factors (VEGF). blood flow with high total renal vascular resistance. The possible mechanism is related to endothelium dysfunction, the hallmark of SSc, with defective response to vasodilation with a tendency towards vasoconstriction (Livi et al., 2002).

In this phase of disease, Doppler ultrasound assessment is a sensitive and non invasive tool to evaluate the renal vascular damage. In aging mice, endostatin has a profibrotic action inducing renal fibrosis. Lin et al. have recently demonstrated in experimental aging animals high levels of endostatin associated with microvascular rarefaction and progressive tubulointerstitial fibrosis (Lin et al., 2014).

On the other hand, intrarenal stiffness is not correlated to VEGF in our study.

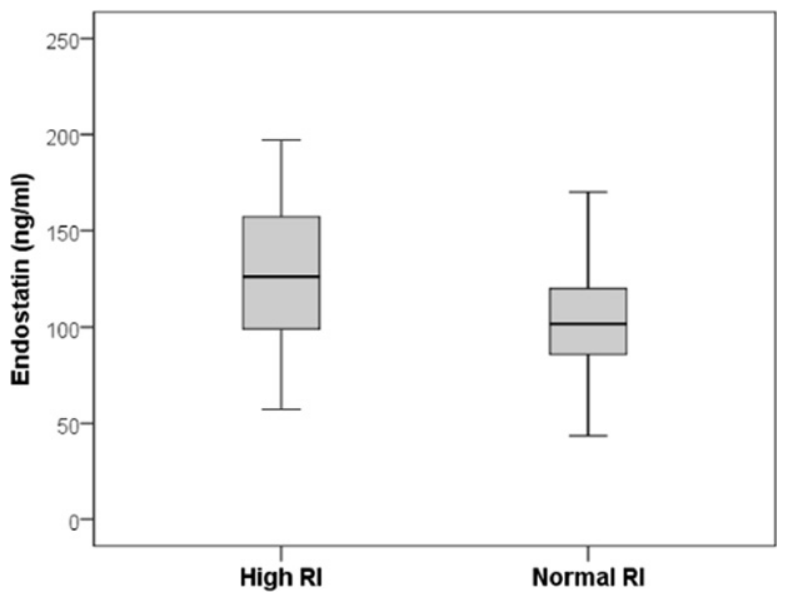

Fig. 2. Serum levels of endostatin (ng/ml) in SSc patients with normal or high RI. 

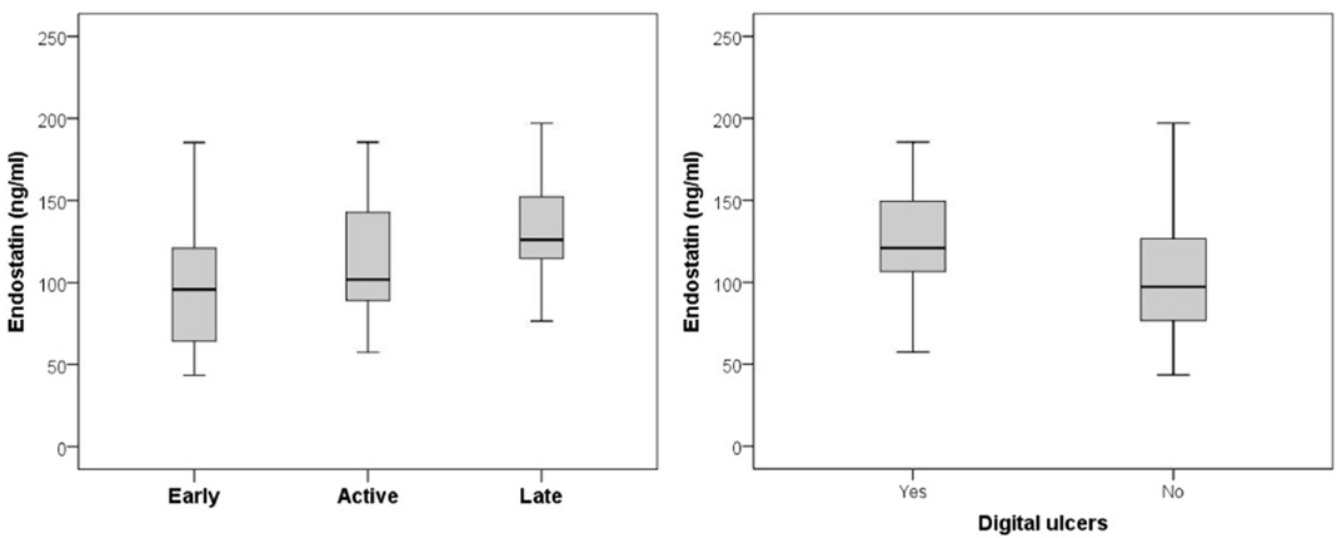

Fig. 3. Serum levels of endostatin (ng/ml) in tree capillaroscopic groups and in SSc patients with or without digital ulcers.

Previously study suggested a biphasic regulation of VEGF. Chronic hypoxia decreases VEGF levels, conversely in acute hypoxia VEGF levels are increased (Olszewska-Pazdrak et al., 2009). In fact several studies confirmed that when renal microcirculation is reduced also VEGF is decreased, as it occurs in chronic glomerulonephritis, ischemic nephropathy, renal artery stenosis, chronic kidney diseases (Chade, 2011).

So, it is not surprising that in our study VEGF does not correlate with intrarenal stiffness and renal function. In our study, the serum levels of endostatin increase with the progression of capillaroscopic damage. In addition, serum levels of endostatin are higher in SSc patients with DUs than in SSc patients without DUs.

To the best of our knowledge, this is the first study that assesses intrarenal hemodynamic parameters and endostatin in SSc patients. As well as in digital damage, endostatin represents a marker of renal scleroderma-associated vasculopathy.

\section{References}

Campbell, P.M., LeRoy, E.C., 1975. Pathogenesis of systemic sclerosis: a vascular hypothesis. Semin. Arthritis Rheum. 4, 351-368.

Chade, A.R., 2011. Renovascular disease, microcirculation, and the progression of renal injury: role of angiogenesis. Am. J. Phys. Regul. Integr. Comp. Phys. 300, R783-R790.

Clements, P., Lachenbruch, P., Siebold, J., White, B., Weiner, S., Martin, R., et al., 1995. Inter and intraobserver variability of total skin thickness score (modified Rodnan TSS) in systemic sclerosis. J. Rheumatol. 22, 1281-1285.

Cutolo, M., Sulli, A., Secchi, M.E., Paolino, S., Pizzorni, C., 2006. Nailfold capillaroscopy is useful for the diagnosis and follow-up of autoimmune rheumatic diseases. A future tool for the analysis of microvascular heart involvement? Rheumatology 45 (Suppl. 4) Oxford. (iv43-6).

Distler, O., Del Rosso, A., Giacomelli, R., Cipriani, P., Conforti, M.L., Guiducci, S., et al., 2002. Angiogenic and angiostatic factors in systemic sclerosis: increased levels of vascular endothelial growth factor are a feature of the earliest disease stages and are associated with the absence of fingertip ulcers. Arthritis Res. 4, R11.

Farouk, H.M., Hamza, S.H., El Bakry, S.A., Youssef, S.S., Aly, I.M., Moustafa, A.A., et al., 2013. Dysregulation of angiogenic homeostasis in systemic sclerosis. Int. J. Rheum. Dis. 16, 448-454.

Futrakul, N., Butthep, P., Laohareungpanya, N., Chaisuriya, P., Ratanabanangkoon, K., 2008. A defective angiogenesis in chronic kidney disease. Ren. Fail. 30, 215-217.

Gigante, A, Rosato, E Massa, R, Rossi, C, Barbano, B., Cianci, R., et al., 2012. Evaluation of Chronic Kidney Disease Epidemiology Collaboration equation to estimate glomerular filtration rate in scleroderma patients. Rheumatology (Oxford) 51, 1426-1431.

Hebbar, M., Peyrat, J.P., Hornez, L., Hatron, P.Y., Hachulla, E., Devulder, B., 2000. Increase concentrations of the circulating angiogenesis inhibitor endostatin in patients with systemic sclerosis. Arthritis Rheum. 43, 889-893.

van den Hoogen, F., Khanna, D., Fransen, J., Johnson, S.R., Baron, M., Tyndall, A., et al., 2013. 2013 classification criteria for systemic sclerosis: an American College of Rheumatology/European League against rheumatism collaborative initiative. Arthritis Rheum. $65,2737-2747$

Hummers, L.K., Hall, A., Wigley, F.M., Simons, M., 2009. Abnormalities in the regulators of angiogenesis in patients with scleroderma. J. Rheumatol. 36, 576-582.
LeRoy, E.C., Black, C., Fleischmajer, R., Jablonska, S., Krieg, T., Medsger Jr., T.A., et al., 1988. Scleroderma (systemic sclerosis): classification, subsets and pathogenesis. J. Rheumatol. 15, 202-205.

Levey, A.S., Stevens, L.A., Schmid, C.H., Zhang, Y.L., Castro 3rd, A.F., Feldman, H.I., et al., 2009. CKD-EPI (Chronic Kidney Disease Epidemiology Collaboration). A new equation to estimate glomerular filtration rate. Ann. Intern. Med. 5 (150), 604-612.

Lin, C.H., Chen, J., Ziman, B., Marshall, S., Maizel, J., Goligorsky, M.S., 2014. Endostatin and kidney fibrosis in aging: a case for antagonistic pleiotropy? Am. J. Physiol. Heart Circ. Physiol. 306, H1692-H1699.

Lin, C.H., Chen, J., Zhang, Z., Johnson, G.V., Cooper, A.J., Feola, J., et al., 2016. Endostatin and transglutaminase 2 are involved in fibrosis of the aging kidney. Kidney Int. 89, $1281-1292$

Livi, R., Teghini, L., Pignone, A., Generini, S., Matucci-Cerinic, M., Cagnoni, M., 2002. Renal functional reserve is impaired in patients with systemic sclerosis without clinical signs of kidney involvement. Ann. Rheum. Dis. 61, 682-686.

Maeshima, Y., Makino, H., 2010. Angiogenesis and chronic kidney disease. Fibrogenesis Tissue Repair 5 (3), 13.

Marek-Trzonkowska, N., Kwieczyńska, A., Reiwer-Gostomska, M., Koliński, T., Molisz, A., Siebert, J., 2015. Arterial hypertension is characterized by imbalance of pro-angiogenic versus anti-angiogenic factors. PLoS One 7 (10), e0126190.

Medsger Jr., T.A., Silman, A.J., Steen, V.D., Black, C.M., Akesson, A., Bacon, P.A., et al., 1999. A disease severity scale for systemic sclerosis: development and testing. J. Rheumatol. 26, 2159-2167.

Mohamed, R.H., Zayed, H.S., Amin, A., 2010. Renal disease in systemic sclerosis with normal serum creatinine. Clin. Rheumatol. 29, 729-737.

Olszewska-Pazdrak, B., Hein, T.W., Olszewska, P., Carney, D.H., 2009. Chronic hypoxia attenuates VEGF signaling and angiogenic responses by downregulation of KDR in human endothelial cells. Am. J. Phys. Cell Phys. 296, C1162-C1170.

Parolini, C., Noce, A., Staffolani, E., Giarrizzo, G.F., Costanzi, S., Splendiani, G., 2009. Renal resistive index and long-term outcome in chronic nephropathies. Radiology 252, 888-889.

Peng, W.J., Yan, J.W., Wan, Y.N., Wang, B.X., Tao, J.H., Yang, G.J., et al., 2012. Matrix metalloproteinases: a review of their structure and role in systemic sclerosis. J. Clin. Immunol. 32, 1409-1414.

Reiseter, S., Molberg Ø., Gunnarsson, R, Lund, M.B., Aalokken, T.M., Aukrust, P, et al, 2015. Associations between circulating endostatin levels and vascular organ damage in systemic sclerosis and mixed connective tissue disease: an observational study. Arthritis Res. Ther. 28 (17), 231.

Rosato, E., Gigante, A., Barbano, B., Cianci, R., Molinaro, I., Rossi, C., et al., 2012. Intrarenal hemodynamic parameters correlate with glomerular filtration rate and digital microvascular damage in patients with systemic sclerosis. Semin. Arthritis Rheum. 41, 815-821.

Ruge, T., Carlsson, A.C., Larsson, T.E., Carrero, J.J., Larsson, A., Lind, L., Ärnlöv, J., 2014. Endostatin level is associated with kidney injury in the elderly: findings from two community-based cohorts. Am. J. Nephrol. 40, 417-424.

Shanmugam, V.K1., Steen, V.D., 2010. Renal manifestations in scleroderma: evidence for subclinical renal disease as a marker of vasculopathy. Int. J. Rheumatol. 2010 (pii: 538589).

Valentini, G., Della Rossa, A., Bombardieri, S., Bencivelli, W., Silman, A.J., D'Angelo, S., et al., 2001. European multicentre study to define disease activity criteria for systemic sclerosis. II. Identification of disease activity variables and development of preliminary activity indexes. Ann. Rheum. Dis. 60, 592-598. 